\title{
CALCULUS FROM THE PAST: MULTIPLE DELAY SYSTEMS ARISING IN CANCER CELL MODELLING
}

\author{
G. C. WAKE ${ }^{\otimes 1}$ and H. M. BYRNE ${ }^{2,3}$ \\ (Received 9 November, 2012; revised 22 January, 2013; first published online 30 April, 2013)
}

\begin{abstract}
Nonlocal calculus is often overlooked in the mathematics curriculum. In this paper we present an interesting new class of nonlocal problems that arise from modelling the growth and division of cells, especially cancer cells, as they progress through the cell cycle. The cellular biomass is assumed to be unstructured in size or position, and its evolution governed by a time-dependent system of ordinary differential equations with multiple time delays. The system is linear and taken to be autonomous. As a result, it is possible to reduce its solution to that of a nonlinear matrix eigenvalue problem. This method is illustrated by considering case studies, including a model of the cell cycle developed recently by Simms, Bean and Koeber. The paper concludes by explaining how asymptotic expressions for the distribution of cells across the compartments can be determined and used to assess the impact of different chemotherapeutic agents.
\end{abstract}

2010 Mathematics subject classification: primary 34K06; secondary 92C37, 34C12.

Keywords and phrases: nonlocal calculus, cell phases, asymptotics.

\section{Introduction}

Nonlocal calculus is a general term used to describe situations (arising in models) where cause and effect are separated explicitly in time (a delay), space, age, or even size, depending on how the cohort or state variables are structured. Arguably the simplest case considered is a time delay where the current evolutionary dynamics depend, in an explicit way, on the state of the system at an earlier time. We consider this situation here, albeit in a system which has many compartments.

\footnotetext{
This is a contribution to the series of invited papers by past ANZIAM medallists (Editorial, issue 52(1)). Graeme Wake was awarded the 2006 ANZIAM medal. Helen Byrne was an Invited Speaker at the ANZIAM 2012 Conference held in Warrnambool, Australia.

${ }^{1}$ Centre for Mathematics in Industry, Institute of Natural \& Mathematical Sciences, Massey University, Private Bag 102-904, North Shore Mail Centre, Auckland 0932, New Zealand;

e-mail: g.c.wake@massey.ac.nz.

${ }^{2}$ Oxford Centre for Collaborative Applied Mathematics, Mathematical Institute, University of Oxford, 24-29 St. Giles', Oxford OX1 3LB, UK; e-mail: Helen.Byrne@maths.ox.ac.uk.

${ }^{3}$ Computational Biology Group, Department of Computer Science, University of Oxford, South Parks Road, Oxford OX1 3QD, UK.

(C) Australian Mathematical Society 2013, Serial-fee code 1446-1811/2013 \$16.00
} 
Models can have point delays or these can be distributed over earlier time intervals. The former have a long history; see, for example, the book by Bellman and Cooke [4]. In the second case, where the models are called distributed delay differential equations (DDDEs) [6, 8], one can often eliminate the nonlocal effect by a simple transformation. Point delays generally resist this and thereby preserve their capacity to simply model complex behaviour. In this paper we focus on point time delays for linear equations. We provide a generic procedure for obtaining exact analytical solutions to equations of this type. By solving the resulting transcendental equations, the solution is then obtained explicitly.

When applied to a multistage model of the cell cycle (details given by Basse et al. [1-3]), our method provides asymptotic expressions for the proportions of cells in the different cell cycle compartments. These results constitute a useful underpinning procedure for evaluating experimentally the effectiveness of potential new drugs for chemical treatment of cancer cells when they are administrated "in vitro" [7].

\section{A simple example}

The following, classic textbook problem illustrates nicely the richness that delay problems exhibit [4]. Let $x(t)$ be a time-dependent variable whose time rate of change at time $t$ depends on its value at a time $T>0$ earlier:

$$
\frac{d x}{d t}=x(t-T)
$$

Delay differential equations of this type are particularly useful when one is seeking a simple formulation for modelling complex phenomena, for example the growth of a population of cells which take, on average, $T$ time units to produce a new offspring.

The spanning set of equation (2.1) is the countably infinite set

$$
\Omega=\left\{e^{\lambda t}: \lambda \in \Lambda\right\}, \quad \text { where } \Lambda=\left\{\lambda: e^{-\lambda T}=\lambda\right\} .
$$

The equation in $\Lambda$ for $\lambda$ has only one real (positive) root $\lambda_{0}$ (with $\lambda_{0}=0.5671$ when $T=1$ ) and an infinite number of complex roots in complex conjugate pairs with negative real part. This enables the problem to be well posed with an arbitrary initial condition

$$
x(t)=x_{0}(t), \quad-T \leq t \leq 0 .
$$

We remark that when $T=0$, the spanning set is one dimensional.

The set $\Lambda$ of $\lambda$ values can be expressed in terms of the Lambert $W$ function, which is defined as the solution of $W(z) e^{W(z)}=z$. If, in equation (2.2), we write $z=\lambda T$, the condition for $z$ is $z e^{z}=T$, and so

$$
z=W_{k}(T), \quad \lambda=\frac{1}{T} W_{k}(T)
$$

where $k=1,2, \ldots$ are the branch values of the Lambert $W$ function. 


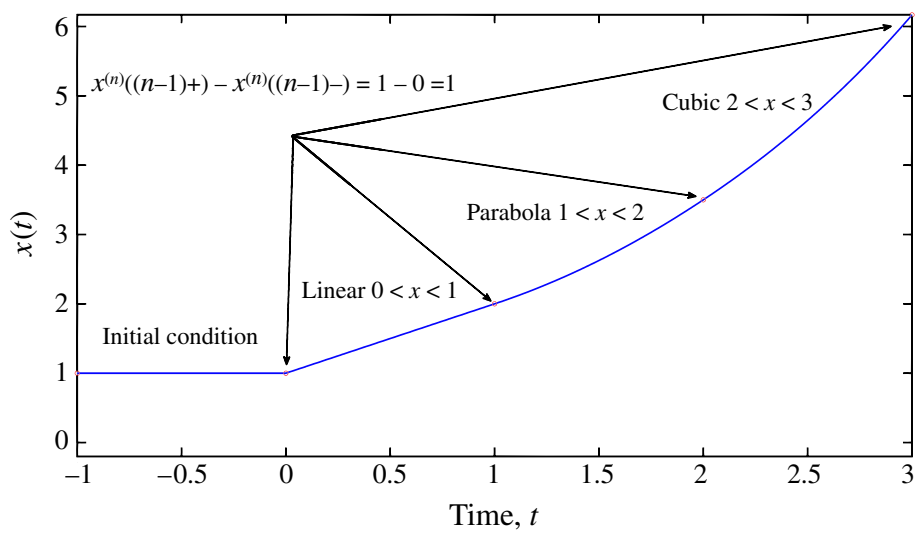

FIGURE 1. Solution of equation (2.1) with unit step time delay $(T=1)$ and constant initial condition $(x(t)=1$ for $-1 \leq t \leq 0)$ obtained by iteration, over successive time intervals.

It is straightforward to deduce the asymptotic behaviour of $x(t)$ :

$$
x(t) \sim K_{0} e^{\lambda_{0} t} \quad \text { for large } t,
$$

where the constant $K_{0}$ depends, in an explicit way, on $x_{0}(t)$. This result, which is easily obtained by Laplace transforms, is far from obvious from the piecewise integration of equation (2.1), which when $T=1$ and $x_{0}(t) \equiv 1$ gives $x(t)$ as a polynomial of degree $n$ in $n T<t<(n+T)$, with discontinuities in higher derivatives as $n$ increases at the end points. This is shown in Figure 1. The pattern here (of the increasing degree of the polynomial as $t$ increases) suggests that the long-term behaviour of $x(t)$ is faster than any power function. However, it does not easily give the value of the exponent in the asymptotic behaviour $\left(x(t) \sim e^{0.57 t}\right.$ for $t$ large $)$.

The general solution of equation (2.1) is obtained by finding the set $\Lambda$ in (2.2). When $T=1$ this gives the transcendental equation $\lambda=e^{-\lambda}$. If we introduce $\lambda_{1}=\operatorname{Re}(\lambda)$ and $\lambda_{2}=\operatorname{Im}(\lambda)$, then $\lambda_{1}$ and $\lambda_{2}$ satisfy the simultaneous equations

$$
\text { (i) } \lambda_{1}=e^{-\lambda_{1}} \cos \left(\lambda_{2}\right), \quad \text { (ii) } \lambda_{2}=-e^{-\lambda_{1}} \sin \left(\lambda_{2}\right) \text {. }
$$

These loci are drawn in Figure 2, with (i) in red and (ii) in blue. The roots are thus the intersections of the red and blue lines.

\section{Multiple delays: a case study}

Multiple delays can arise naturally in many systems. In Section 3.1 we introduce an example based on a model for cell cycle population dynamics due to Simms et al. [10]. We now give the general method for solving such a system explicitly. This is abstracted and written as

$$
\frac{d \mathbf{x}}{d t}=\mathbf{A x}(t-[\mathbf{T}])
$$




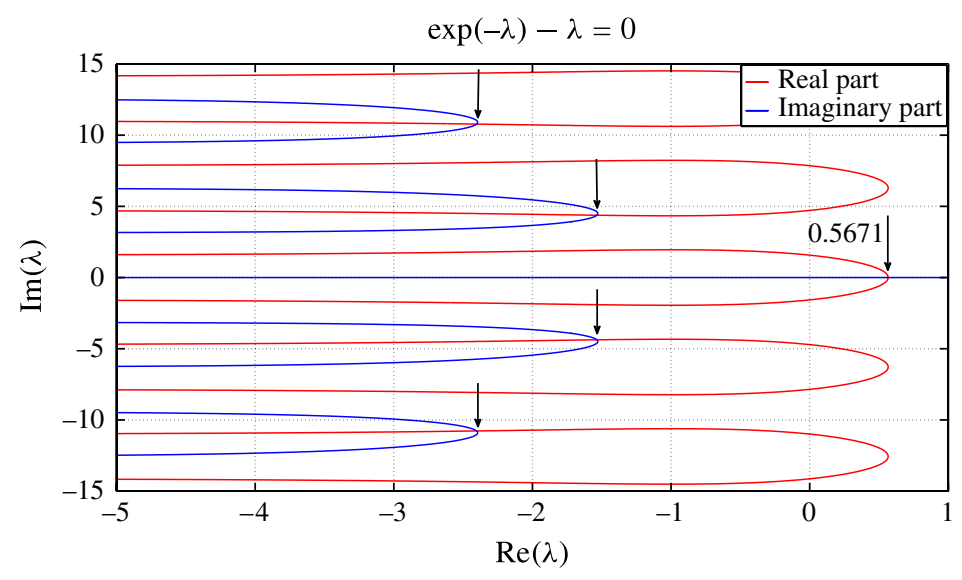

FIGURE 2. The roots of the transcendental equations (2.3). The only roots shown are $\lambda=0.5671$, $-1.543 \pm 4.375 i,-2.402 \pm 10.776 i$. The real part is shown in red and the imaginary part in blue.

where $\mathbf{x}(t)$ is a column vector of functions $\left\{x_{1}(t), \ldots, x_{i}(t), \ldots, x_{n}(t)\right\}, \mathbf{A}$ is an $n \times n$ (constant) matrix, $\mathbf{T}=\left(T_{i j}\right)$ and $[\mathbf{T}]$ denotes $T_{i j}$ when $\mathbf{x}$ is replaced by $x_{i}$ to write (3.1) in component form; that is,

$$
\frac{d x_{i}}{d t}=\sum_{j} a_{i j} x_{j}\left(t-T_{i j}\right) .
$$

To solve equation (3.2) we let $\mathbf{x}(t)=\exp (\lambda t) \mathbf{c}$, where $\mathbf{c}$ is a constant eigenvector and $\lambda$ the corresponding eigenvalue. This was the form of the solution used by Hale et al. [5], whose main focus was to determine the effect of the delays on the stability of the trivial solution. Here we extend that system [5] to the case where there is a matrix of delays rather than a vector, and we determine the stability (as Hale et al. did) by the existence of a value of $\lambda$ for which $\operatorname{Re}(\lambda)>0$.

We then have $\lambda \mathbf{c}=\mathbf{B}(\lambda) \mathbf{c}$, where $\mathbf{B}(\lambda)=\left[a_{i j} e^{-\lambda T_{i j}}\right]$. So

$$
\lambda \in\{\lambda: \operatorname{det}(\mathbf{B}(\lambda)-\lambda I)=0\}=\mathbf{\Lambda} .
$$

This is a "nonlinear in $\lambda$ " eigenvalue problem with a countably infinite number of "eigenvalues" and associated eigenvectors $\mathbf{c}(\lambda)$. The general solution of $d \mathbf{x} / d t=$ $\mathbf{A x}(t-[\mathbf{T}])$ is, therefore,

$$
\mathbf{x}(t)=\sum_{\lambda \in \boldsymbol{\Lambda}} e^{\lambda t} \mathbf{c}(\lambda) .
$$

We note the following properties which are inferred from examples only:

- If $T_{i j} \geq 0$ (as in the tumour model below) for all $i, j$ then there are a finite number of real eigenvalues which are positive, and all others (an infinite number) are complex, occurring in conjugate pairs, with smaller real part. 


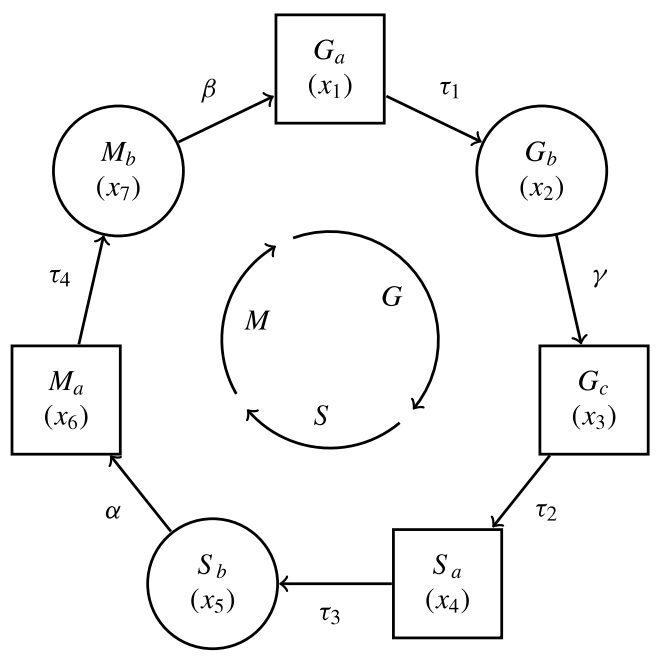

FIGURE 3. Schematic of the cell cycle model developed by Simms et al. [9, 10], showing how cells progress through the seven model phases. Each phase is associated with a specific cell cycle phase (inner cyclic arrows). Square boxes are used to denote storage phases, while circles represent nonstorage phases. The parameters on arrows from storage phases represent the fixed time that a cell spends in that model phase, while the parameters on arrows from nonstorage phases represent the rates of transition from those phases.

- Since the number of real and positive eigenvalues is finite, we deduce the asymptotic behaviour $\mathbf{x}(t) \sim e^{\lambda_{0} t} \mathbf{c}\left(\lambda_{0}\right)$ as $t \rightarrow \infty$, where $\lambda_{0}$ is the "eigenvalue" with largest real part.

- Consequently, $x_{i}(t) / x_{j}(t) \sim c_{i} / c_{j}$ as $t \rightarrow \infty$, that is, the proportions evolve to constants at long times. This result will be useful when we consider the response of tumours to chemotherapy.

3.1. Model development Mathematical models often decompose the cell cycle into three distinct phases: the G1 (gap, written as G), S (DNA replication) and G2/M (gap/mitosis, written as M) phases. In the model of Simms et al. [9, 10], these phases are further subdivided into storage and nonstorage phases, the latter being ones in which the cells are responsive to their environment. Storage phases are ones in which the cells stay for a fixed time. A schematic of the Simms et al. model is presented in Figure 3, reproduced from their paper [10].

We introduce $N_{G_{a}}(t)=x_{1}(t), N_{G_{b}}(t)=x_{2}(t), \ldots, N_{M_{b}}(t)=x_{7}(t)$ to represent the number of cells in the $G_{a}, G_{b}, \ldots, M_{b}$ phases at time $t$, respectively, and recall that $d X(t) / d t$ represents the time rate of change of $X(t)$. Referring to Figure 3, we deduce that the model equations may be written as follows:

$$
\begin{aligned}
& \frac{d x_{1}}{d t}=2 \beta x_{7}(t)-2 \beta x_{7}\left(t-\tau_{1}\right), \\
& \frac{d x_{2}}{d t}=2 \beta x_{7}\left(t-\tau_{1}\right)-\gamma x_{2}(t),
\end{aligned}
$$




$$
\begin{aligned}
& \frac{d x_{3}}{d t}=\gamma x_{2}(t)-\gamma x_{2}\left(t-\tau_{3}\right), \\
& \frac{d x_{4}}{d t}=\gamma x_{2}\left(t-\tau_{3}\right)-\gamma x_{2}\left(t-\tau_{3}-\tau_{2}\right), \\
& \frac{d x_{5}}{d t}=\gamma x_{2}\left(t-\tau_{3}-\tau_{2}\right)-\alpha x_{5}(t), \\
& \frac{d x_{6}}{d t}=\alpha x_{5}(t)-\alpha x_{5}\left(t-\tau_{4}\right), \\
& \frac{d x_{7}}{d t}=\alpha x_{5}\left(t-\tau_{4}\right)-\beta x_{7}(t) .
\end{aligned}
$$

The parameters $\alpha, \beta, \gamma$ represent rate constants for movement from the nonstorage phases/compartments into the storage compartments. The factor " 2 " indicates cell division into two daughter cells. All these parameters are taken as constant, making our system autonomous. The $\tau$ values are the fixed times the cells stay in the four storage phases (see Figure 1). These equations are of the general form presented in equation (3.1). Consequently, in the subsections that follow we apply the method from the first part of Section 3 to study this problem.

3.2. Model simplification and analysis Scrutiny of the equations (3.3)-(3.9) reveals that the four storage phases $x_{1}, x_{3}, x_{4}, x_{6}$ decouple and, hence, that the system is effectively driven by a smaller, three-dimensional system that comprises equations (3.4), (3.7) and (3.9). Following the approach outlined at the beginning of Section 3, it is straightforward to show that the "eigenvalues" satisfy the transcendental equation

$$
\lambda^{3}+(\alpha+\beta+\gamma) \lambda^{2}+(\alpha \beta+\beta \gamma+\gamma \alpha) \lambda+\alpha \beta \gamma\left(1-2 e^{-\lambda\left(\tau_{1}+\tau_{2}+\tau_{3}+\tau_{4}\right)}\right)=0 .
$$

Equation (3.10) could easily be solved (numerically) and the corresponding eigenvectors obtained. However, we do not do this here as we are interested in determining the fraction of cells in each of the seven compartments (including the storage ones) and this can be accomplished for the full system in one step. We observe that $\lambda=0$ is not a solution of equations (3.3)-(3.9), showing that there are no constant solutions of this core solution. Further, there is just one real solution which is positive, and it will drive the dynamics of the solution in the long term if the system is not perturbed. This is clarified in the next section where all of the equations are included.

3.3. Analysis of the full system Here we use the method from the beginning of Section 3 to solve the full model as defined by equations (3.3)-(3.9). It is, however, important to note that the transcendental equation arising from (3.3)-(3.9) will be the same as that in (3.10), apart from the fact that $\lambda=0$ will be an "eigenvalue" of geometric multiplicity four, showing that there are constant solutions (not growing in time) as well as possibly some with slower (algebraic) growth in time. Secondly, by writing $X=\sum_{i} x_{i}$ for the total number of cells, we see on adding the seven equations (3.3)-(3.9) that

$$
\frac{d X}{d t}=\beta x_{7}
$$


TABLE 1. Parameters from the cancer cell lines in the paper by Simms et al. [10].

\begin{tabular}{ll}
\hline Symbol & Value and units \\
\hline$\alpha$ & $0.6700 \mathrm{~h}^{-1}$ \\
$\beta$ & $2.3100 \mathrm{~h}^{-1}$ \\
$\gamma$ & $0.3284 \mathrm{~h}^{-1}$ \\
$\tau_{1}$ & $5.75 \mathrm{~h}$ \\
$\tau_{2}$ & $1.00 \mathrm{~h}$ \\
$\tau_{3}$ & $9.00 \mathrm{~h}$ \\
$\tau_{4}$ & $3.40 \mathrm{~h}$ \\
\hline
\end{tabular}

So the total number of cells mirrors that of its constituent components with the same asymptotic behaviour.

Before proceeding to solve the full system, we observe that some of the components have more than one delay. For example, $x_{2}$ appears in the governing equations evaluated at time $t$ (that is, with no delay) and also evaluated at times $t-\tau_{3}$ and $t-\tau_{3}-\tau_{2}$ (two different delays). We therefore need to adapt the method above to account for more general systems of the form

$$
\frac{d \mathbf{x}}{d t}=\mathbf{A}^{(1)} \mathbf{x}\left(t-\left[\mathbf{T}^{(1)}\right]\right)+\mathbf{A}^{(2)} \mathbf{x}\left(t-\left[\mathbf{T}^{(2)}\right]\right)+\mathbf{A}^{(3)} \mathbf{x}\left(t-\left[\mathbf{T}^{(3)}\right]\right) .
$$

That is, there are three different "matrices of delay times", $\mathbf{T}^{(k)}, k=1,2,3$. Values from the experimental situation [10] are given in Table 1. This gives the six, very sparse, $7 \times 7$ matrices below:

$$
\begin{aligned}
\mathbf{A}^{(1)}=\left[\begin{array}{ccccccc}
0 & 0 & 0 & 0 & 0 & 0 & 2 \beta \\
0 & -\gamma & 0 & 0 & 0 & 0 & 0 \\
0 & \gamma & 0 & 0 & 0 & 0 & 0 \\
0 & 0 & 0 & 0 & 0 & 0 & 0 \\
0 & 0 & 0 & 0 & -\alpha & 0 & 0 \\
0 & 0 & 0 & 0 & \alpha & 0 & 0 \\
0 & 0 & 0 & 0 & 0 & 0 & -\beta
\end{array}\right], \quad \mathbf{T}^{(1)}=\left[\begin{array}{ccccccc}
0 & 0 & 0 & 0 & 0 & 0 & 0 \\
0 & 0 & 0 & 0 & 0 & 0 & 0 \\
0 & 0 & 0 & 0 & 0 & 0 & 0 \\
0 & 0 & 0 & 0 & 0 & 0 & 0 \\
0 & 0 & 0 & 0 & 0 & 0 & 0 \\
0 & 0 & 0 & 0 & 0 & 0 & 0 \\
0 & 0 & 0 & 0 & 0 & 0 & 0
\end{array}\right]=\mathbf{0} \\
\mathbf{A}^{(2)}=\left[\begin{array}{ccccccc}
0 & 0 & 0 & 0 & 0 & 0 & -2 \beta \\
0 & 0 & 0 & 0 & 0 & 0 & 2 \beta \\
0 & -\gamma & 0 & 0 & 0 & 0 & 0 \\
0 & \gamma & 0 & 0 & 0 & 0 & 0 \\
0 & 0 & 0 & 0 & 0 & 0 & 0 \\
0 & 0 & 0 & 0 & -\alpha & 0 & 0 \\
0 & 0 & 0 & 0 & \alpha & 0 & 0
\end{array}\right], \quad \mathbf{T}^{(2)}=\left[\begin{array}{ccccccc}
0 & 0 & 0 & 0 & 0 & 0 & \tau_{1} \\
0 & 0 & 0 & 0 & 0 & 0 & \tau_{1} \\
0 & \tau_{3} & 0 & 0 & 0 & 0 & 0 \\
0 & \tau_{3} & 0 & 0 & 0 & 0 & 0 \\
0 & 0 & 0 & 0 & 0 & 0 & 0 \\
0 & 0 & 0 & 0 & \tau_{4} & 0 & 0 \\
0 & 0 & 0 & 0 & \tau_{4} & 0 & 0
\end{array}\right],
\end{aligned}
$$




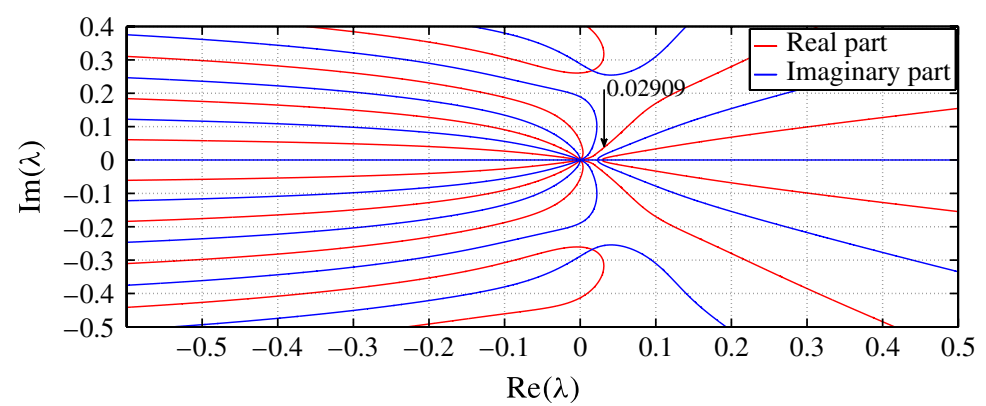

FIGURE 4. Loci for $F(\lambda)=\operatorname{det}(\mathbf{B}(\lambda)-\lambda I)=0$. The real parts are shown in red and the imaginary part in blue.

$$
\mathbf{A}^{(3)}=\left[\begin{array}{ccccccc}
0 & 0 & 0 & 0 & 0 & 0 & 0 \\
0 & 0 & 0 & 0 & 0 & 0 & 0 \\
0 & 0 & 0 & 0 & 0 & 0 & 0 \\
0 & -\gamma & 0 & 0 & 0 & 0 & 0 \\
0 & \gamma & 0 & 0 & 0 & 0 & 0 \\
0 & 0 & 0 & 0 & 0 & 0 & 0 \\
0 & 0 & 0 & 0 & 0 & 0 & 0
\end{array}\right], \quad \mathbf{T}^{(3)}=\left[\begin{array}{ccccccc}
0 & 0 & 0 & 0 & 0 & 0 & 0 \\
0 & 0 & 0 & 0 & 0 & 0 & 0 \\
0 & 0 & 0 & 0 & 0 & 0 & 0 \\
0 & \tau_{2}+\tau_{3} & 0 & 0 & 0 & 0 & 0 \\
0 & \tau_{2}+\tau_{3} & 0 & 0 & 0 & 0 & 0 \\
0 & 0 & 0 & 0 & 0 & 0 & 0 \\
0 & 0 & 0 & 0 & 0 & 0 & 0
\end{array}\right] .
$$

From the corresponding equation

$$
F(\lambda)=\operatorname{det}(\mathbf{B}(\lambda)-\lambda I)=0,
$$

where

$$
\mathbf{B}(\lambda)=\sum_{k=1}^{3}\left(A_{i j}^{(k)} e^{-\lambda T_{i j}^{(k)}}\right)
$$

we obtain the diagram in Figure 4. So $\mathbf{x} \sim e^{\lambda_{\max t}} \mathbf{c}$, with $\lambda_{\max }=0.02909 \mathrm{~h}^{-1}$. Now we just need to find $\mathbf{c}=\left(c_{1}, \ldots, c_{7}\right)$ such that

$$
\mathbf{B}\left(\lambda_{\max }\right) \mathbf{c}=\lambda_{\max } \mathbf{c} .
$$

In this cell-growth case study, the relative signs of the real parts of the roots of the equation for $\lambda$ give an indication of the rate of approach to the asymptotic form. In Figure 4, we see that the second eigenvalues $\lambda_{2}$ (a complex conjugate pair) have positive real part and are the only such ones, in fact, along with $\lambda_{\max }$. So the approach to the solution above will be relatively slow but it will happen as

$$
\mathbf{x}(t) \sim e^{\lambda_{\max } t} \mathbf{c}+e^{\lambda_{2} t} \mathbf{c}^{(2)} \sim e^{\lambda_{\max } t}\left(\mathbf{c}+e^{\left(\lambda_{2}-\lambda_{\max }\right) t} \mathbf{c}^{(2)}\right),
$$

where $\mathbf{c}^{(2)}$ is an eigenvector association with the "eigenvalue" $\lambda_{2}$. Thus the time constant of the approach to that of the basic exponential growth, with constant proportions given by the elements of the vector $\mathbf{c}$, is $\operatorname{Re}\left(\lambda_{2}\right)-\lambda_{\max }<0$. 
Equation (3.13) gives

$$
\mathbf{c}=\left[\begin{array}{l}
0.0442 \\
0.1218 \\
0.4380 \\
0.2680 \\
0.0373 \\
0.0809 \\
0.0097
\end{array}\right], \quad \text { corresponding to the phases }\left[\begin{array}{c}
G_{a} \\
G_{b} \\
G_{c} \\
S_{a} \\
S_{b} \\
M_{a} \\
M_{b}
\end{array}\right]
$$

from Figure 3. That is, in the long term, the cell proportions are approximately $0.0442+0.1218+0.4380=60.3 \%$ in the $\mathrm{G}$ phase, $30.1 \%$ in the $\mathrm{S}$ phase, and $9.1 \%$ in the $\mathrm{M}$ phase. The solution vector is

$$
\mathbf{x}(t) \sim(0.0442,0.1218,0.4380,0.2680,0.0373,0.0809,0.0097) e^{\rho t},
$$

where $\rho=0.029 \mathrm{~h}^{-1}$. This approximate solution gives the asymptotic proportion of cells in each compartment at long times.

\section{Conclusion}

We have presented an explicit method for solving systems of the form (3.1) and extensions of the form (3.11). The method produces a countable number of solutions which span the set of all solutions. When the dimension of the system is odd (here 7 or 3 ) there is one real positive time constant that is bigger than the real parts of any other solutions for $\lambda$ in equation (3.12) and dominates the long-term behaviour of the solution. This has neglected the effects of logistic inputs. The complex roots of $F(\lambda)=$ 0 give damped oscillatory behaviour. This seemingly simple example illustrates the fact that delay and especially multiple-delay systems can exhibit complex behaviour.

\section{Acknowledgements}

This paper is based on work supported in part by Award No. KUK-C1-013-04 made by King Abdullah University of Science and Technology (KAUST). Thanks are due to Mr. Ali Ashher Zaidi, Ph.D. candidate at Massey University, for the software for the diagrams in Figures 2 and 4, and research assistant Miss Andrea Babylon for technical assistance. We also thank Dr. Kate Simms and her co-workers as well as Springer-Verlag for permission to reproduce Figure 3.

\section{References}

[1] B. Basse, B. C. Baguley, E. S. Marshall, W. R. Joseph, B. van Brunt, G. C. Wake and D. J. N. Wall, "A mathematical model for analysis of the cell cycle in cell lines derived from human tumors", J. Math. Biol. 47 (2003) 295-312; doi:10.1007/s00285-003-0203-0.

[2] B. Basse, B. C. Baguley, E. S. Marshall, W. R. Joseph, B. van Brunt, G. C. Wake and D. J. N. Wall, "Modelling cell death in human tumour cell lines exposed to the anticancer drug paclitaxel", J. Math. Biol. 49 (2004) 329-357; doi:10.1007/s00285-003-0254-2. 
[3] B. Basse, B. C. Baguley, E. S. Marshall, G. C. Wake and D. J. N. Wall, "Modelling the flow of cytometric data obtained from unperturbed human tumour cell lines: parameter fitting and comparison", Bull. Math. Biol. 67 (2005) 815-830; doi:10.1016/j.bulm.2004.10.003.

[4] R. Bellman and K. L. Cooke, Differential-difference equations (Academic Press, New York, 1963).

[5] J. K. Hale, E. F. Infante and F.-S. P. Tsen, "Stability in linear delay equations", J. Math. Anal. Appl. 105 (1985) 533-555; doi:10.1016/0022-247X(85)90068-X.

[6] H. V. Jain and H. M. Byrne, "Qualitative analysis of an integro-differential model of periodic chemotherapy", Appl. Math. Lett. 25 (2012) 2132-2136; doi:10.1016/j.aml.2012.04.024.

[7] L. A. Johnson, H. M. Byrne, A. E. Willis and C. A. Laughton, "An integrative biological approach to the analysis of tissue culture data: application to the anti-tumour agent RHPS4", Integr. Biol. 3 (2011) 843-849; doi:10.1039/C1IB00025J.

[8] H. Rasmussen, G. C. Wake and J. Donaldson, "Analysis of a class of distributed delay logistic differential equations", Math. Comput. Model. 38 (2003) 123-132; doi:10.1016/S0895-7177(03)90010-0.

[9] K. Simms, "A mathematical model of cell cycle progression applied to breast cancer cell lines", $\mathrm{Ph}$. D. Thesis, University of Adelaide, 2011.

[10] K. Simms, N. Bean and A. Koerber, "A mathematical model of cell cycle progression applied to the MCF-7 breast cancer cell line", Bull. Math. Biol. 74 (2012) 736-767;

doi:10.1007/s11538-011-9700-2. 